\title{
Diversidade de insetos em plantas hospedeiras próximas às áreas de cultivo de milho e algodão
}

\author{
Rafael Alvim Gonzaga de Oliveira', Jael Simões Santos Rando \\ ${ }^{1}$ Universidade Estadual do Norte do Paraná - UENP, Campus Luiz Meneghel, Bandeirantes, Paraná, Brasil. E-mail: \\ rafael.ago@gmail.com, jael@uenp.edu.br
}

Recebido: 10/04/2017; Aceito: 27/06/2017.

\section{RESUMO}

As plantas hospedeiras servem como refúgios para os insetos no período de entressafra, onde se alimentam, acasalam ou ficam em diapausa aguardando novo plantio para que os indivíduos sobreviventes se dirijam à lavoura. Este estudo teve como objetivo avaliar a diversidade de insetos em treze espécies de plantas espontâneas próximas a áreas de cultivo de algodão e milho no período de entressafra. Foram selecionadas quatro plantas hospedeiras e realizadas avaliações mensais por observação direta e identificados os insetos. Foi realizada a análise faunística determinando-se a riqueza, índice de diversidade de Margalef e Shannon-Wiener e índice de equitabilidade. Foram coletados indivíduos pertencentes às ordens Coleoptera, Hemiptera, Hymenoptera, Diptera, Lepidoptera e Neuroptera, tendo com maior incidência a praga Diabrotica speciosa. Entre as plantas hospedeiras próximas a cultura do algodão foram encontradas as ordens de insetos Coleoptera, Hemiptera, Hymenoptera, Diptera e Neuroptera, sendo Dysdercus ruficollis a principal representante. Os índices faunísticos mais elevados foram verificados nas plantas Coniza sp. e Cenchrus echinatus próximas a área de cultivo de milho e Commelina bengalensis e Parthenium hysterophorus nas plantas próximas a área de cultivo de algodão.

Palavras-chave: Gossypium hirsutum, Insecta, plantas espontâneas, refúgio, Zea mays

\section{Diversity of insects in host plants near cultivation areas of corn and cotton}

\begin{abstract}
Host plants serve as refuge for insects in off-season, where they feed, breed or stay in diapause and when there is a new crop, surviving subjects go to the crop. With the objective of evaluate the diversity of insects in thirteen species of spontaneous plants next to fields of cotton and corn cultivation in the off-season they were selected four subjects from each plant and conducted monthly evaluations by direct observation, insect collected and identified. It was performed faunistic analysis determining wealth, diversity and evenness indexes. They were collected subjects belonging Coleoptera, Hemiptera, Hymenoptera, Diptera, Lepidoptera and Neuroptera orders happening with higher incidence of Diabrotica speciosa. Among host plants next to field of cotton cultivation were obtained Coleoptera, Hemiptera, Hymenoptera, Diptera and Neuroptera orders, with Dysdercus ruficollis the maine representative. Higher indexes verified in plants located near by the field of corn cultivation was in Coniza sp. and Cenchrus echinatus and Commelina bengalensis and Parthenium hysterophorus in plants next the field of cotton cultivation.
\end{abstract}

Key words: Gossypium hirsutum, Insecta, spontaneous plants, refuge, Zea mays. 


\section{Introdução}

Fragmentos florestais ou plantas hospedeiras nas proximidades das áreas de cultivo servem como refúgios, para que não ocorra interrupção do ciclo de vida dos insetos. De acordo com Godoy et al. (2010) no período de entressafra, os insetos deslocam-se para abrigos de sobrevivência permanecendo nesses locais em diapausa.

Plantas hospedeiras silvestres podem se constituir em ambiente favorável para desenvolvimento de populações de percevejos como os pentatomideos que as utilizam como fonte de alimentação e reprodução para infestar as plantas cultivadas (PANIZZI et al., 2012).

Por serem hospedeiras de insetos praga e patógenos (ALTIERI et al., 2003) e por interferirem no crescimento vegetativo das culturas por meio da liberação de substâncias químicas (RICE, 1974), competição por água, luz, nutrientes, (FUERST; PUTNAN, 1983) e cobertura (FAVERO et al., 2001), as plantas hospedeiras, também conhecidas como plantas invasoras ou daninhas, podem ser consideradas plantas indesejáveis.

Mas segundo Macedo e Martins (1998), se mantidas em locais estratégicos, as plantas hospedeiras podem ser extremamente benéficas. Sua manutenção em agroecossistemas pode diminuir de maneira significativa as populações de espécies pragas, devido ao aumento da diversidade e abundância de inimigos naturais (ALTIERI et al., 2003).

A manutenção de plantas hospedeiras pode favorecer a diversificação da entomofauna associada, pois segundo Carrano-Moreira (2014), aumentar a diversidade de plantas significa diversificar a fauna diminuindo o fluxo de energia do ecossistema aumentando sua estabilidade.

Este estudo teve como objetivo avaliar a diversidade dos insetos pragas e inimigos naturais em espécies de plantas hospedeiras próximas a áreas cultivadas com algodão e milho no período de entressafra

\section{Material e Métodos}

O trabalho foi conduzido, nos meses de agosto a novembro de 2015 no município de Bandeirantes- PR, localizado à $23^{\circ} 06^{\prime} 36^{\prime \prime}$ Sul, 50²2'03" Oeste e altitude de 420m (IBGE 2010). Tomou-se como referência, para fins de pesquisa, duas culturas anuais de verão, o algodão em pleno desenvolvimento reprodutivo ocupando uma área de $2200 \mathrm{~m}^{2}$ e a cultura do milho, em período vegetativo numa área de $1000 \mathrm{~m}^{2}$.

Inicialmente, próximo a essas culturas, foi feita a seleção, identificação e demarcação de quatro indivíduos de todas as plantas hospedeiras. Os tratamentos foram constituídos por treze espécies de plantas hospedeiras: $\mathrm{T}_{1}$ buva (Coniza sp.); $\mathrm{T}_{2}$ capimcarrapicho (Cenchrus echinatus); $\mathrm{T}_{3}$ caruru (Amaranthus sp.); $\mathrm{T}_{4}$ amendoim-bravo (Euphorbia heterophylla); $\mathrm{T}_{5}$ nabiça (Raphanus raphanistrum); $\mathrm{T}_{6}$ picão-preto (Bidens pilosa); $\mathrm{T}_{7}$ serralha (Sonchus oleraceus) e distavam entre $1 \mathrm{~m}$ a $3 \mathrm{~m}$ da área de milho. Distando de $5,5 \mathrm{~m}$ a $25 \mathrm{~m}$ do algodão os tratamentos: $\mathrm{T}_{8}$ capim amargoso (Digitaria insularis); $\mathrm{T}_{9}$ capim colonião (Panicum maximum); $\mathrm{T}_{10}$ guanxuma (Sida $\mathrm{sp}$.); $\mathrm{T}_{11}$ losna-branca (Parthenium hysterophorus); $\mathrm{T}_{12}$ rubim (Leonurus sibiricus) e $\mathrm{T}_{13}$ trapoeraba (Commelina benghalensis). Admitiu-se como amostra uma planta hospedeira por tratamento, sendo utilizadas quatro repetições.

Foram realizadas avaliações mensais e optou-se pela observação direta ou exame visual individualizado, o que permite avaliar a planta inteira sem danificá-la. Tal método também permite a observação de ovos e pupas (CORRÊA-FERREIRA, 2012). Os insetos coletados foram preservados em álcool $70 \%$ e identificados por entomologistas.

Foi realizada a análise faunística determinando-se a riqueza de espécies (S), índices de diversidade de Margalef $(\alpha)$ e Shannon-Wiener $(H)$ e o Índice de equitabilidade (E). O índice de diversidade de Margalef $(\alpha)$; a relação entre o número de espécie e o número total de indivíduos das espécies presente na comunidade foi calculado segundo a equação citada por Southwood (1995):

$$
\alpha=(S-1) / \operatorname{Ln}(N)
$$

onde, $\alpha=$ indice de diversidade de Margalef, $S=$ número de espécies amostrado, Ln = logaritmo de base R. raphanistrum neperiana, $N=$ número de indivíduos coletados.

O Índice de diversidade de Shannon-Wiener $(\mathrm{H})$ foi calculado segundo a equação citada por Ricklefs (1996).

$$
H=-\sum[(n / N) * \operatorname{Ln}(n / N)]
$$

onde, $H$ = indice de Shannon-Wiener, $n=$ número de indivíduos, Ln = logaritmo de base neperiana, $N=$ número de espécies.

Segundo Pinto-Coelho (2000), a equitabilidade representa a uniformidade do número de indivíduos entre as espécies, com valor variado de 0 a 1 , que é a razão entre o índice de diversidade Shannon-Wiener e a diversidade máxima, a qual parte do pressuposto que todas as espécies tem a mesma abundância.

$$
E=H / \operatorname{Ln}(S)
$$

onde, $E$ = equitabilidade, $H=$ indice de ShannonWiener, Ln = logaritimo de base neperiana, $S=$ número de espécies. 


\section{Resultados e Discussão}

Foram coletados nas plantas hospedeiras próximas a cultura do milho 232 espécimes de insetos pertencentes às ordens Coleoptera, Hemiptera, Hymenoptera, Diptera, Lepidoptera e Neuroptera. Dessas, a ordem Coleoptera respondeu por $46,55 \%$ dos insetos praga ou de natureza predatória. Na família Chrysomelidae, que compreende besouros desfolhadores, Diabrotica speciosa (Germar) esteve presente em todas as plantas hospedeiras, exceto em Coniza sp. (Tabela 1).

Os adultos de D. speciosa são polífagos, alimentamse de folhas, brotações novas, vagens e frutos de várias espécies de plantas de importância econômica (SALVADORI et. al., 2004), vulgarmente conhecida como vaquinha-verde-amarela, patriota ou brasileirinho é amplamente distribuída nos estados brasileiros e em alguns países da América do Sul.

A cigarrinha Ceresa brunnicornis (L.) com ocorrência recente em soja (HOFFMANN-CAMPO et al., 2012) foi a única praga registrada na planta daninha Coniza sp. Plantas desse gênero são popularmente conhecidas por "buva" ou "voadeira" e têm-se destacado desde a década de 1990 por infestarem áreas abandonadas (terrenos baldios e margens de estradas), pastagens, culturas perenes como citros e café e lavouras anuais como algodão, milho, soja e trigo (THEBAUD; ABBOTT, 1995).

A planta daninha Sonchus oleraceus foi a que hospedou o maior número de insetos, sendo o pulgão Uroleucon ambrosiae (Thomas) o mais numeroso. Os afídeos dessa espécie são relativamente grandes, com tamanho de 3,0 a 3,5 mm, de coloração vermelha escura e formato alongado; fáceis de serem distinguidos nas plantas. Embora também se alimentem nas folhas tem certa preferência pelas hastes floríferas. Ocorrem com frequência nas plantas silvestres da família Asteraceae (AUAD et al., 2003).

Destacaram-se como inimigos naturais cinco espécies de Coccinelidae. Esses besouros conhecidos por "joaninhas" embora se alimentem preferencialmente de pulgões predam também ácaros, cochonilhas e tripes (NDAKIDEMI et al., 2016). A espécie Eriopis connexa (Ger.) foi a mais numerosa, provavelmente devido à variedade de presas. A polifagia de Eriopis connexa foi relatada por Silva et al. (2013) sugerindo que essa espécie pode se alimentar de um grande número de pragas de milho e sorgo, e que a disponibilidade e qualidade de alimentos são fundamentais para estabelecimento da espécie em uma nova área.

Tabela 1 - Número total de espécies de insetos (t), coletados próximo à área cultivada com milho nas plantas hospedeiras, localizadas à distância de $\mathrm{T}_{1}$ Coniza sp. $(2 \mathrm{~m}) ; \mathrm{T}_{2}$ C. echinatus $(1 \mathrm{~m}) ; \mathrm{T}_{3}$ Amaranthus spp. $(2,8 \mathrm{~m}) ; \mathrm{T}_{4}$ E. heterophylla $(2,5 \mathrm{~m}) ; \mathrm{T}_{5} R$. raphanistrum $(2,3 \mathrm{~m}) ; \mathrm{T}_{6}$ B. pilosa $(2,6 \mathrm{~m})$ e $\mathrm{T}_{7} S$. oleraceus $(1,6 \mathrm{~m})$, de área com cultivo. Bandeirantes, $\mathrm{PR}$.

\begin{tabular}{|c|c|c|c|c|c|c|c|c|c|}
\hline \multirow{2}{*}{ Ordem } & \multirow{2}{*}{ Insetos } & \multicolumn{8}{|c|}{ Plantas hospedeiras } \\
\hline & & $\mathrm{T}_{1}$ & $\mathrm{~T}_{2}$ & $\mathrm{~T}_{3}$ & $\mathrm{~T}_{4}$ & $\mathrm{~T}_{5}$ & $\mathrm{~T}_{6}$ & $\mathrm{~T}_{7}$ & $\mathrm{t}$ \\
\hline \multirow{9}{*}{ Coleoptera } & Astylus variegatus (Germar) & - & - & - & - & 1 & - & - & 1 \\
\hline & Cerotoma arcuata (Olivier) & 4 & - & - & - & - & - & - & 4 \\
\hline & Diabrotica speciosa (Germar) & - & 2 & 12 & 5 & 15 & 5 & 2 & 41 \\
\hline & Lagria villosa (Fabricius) & 2 & 1 & - & - & - & - & - & 3 \\
\hline & Cycloneda sanguinea (Linnaeus) & - & 2 & - & - & 1 & 2 & - & 5 \\
\hline & Eriopis connexa (Germar) & 3 & 1 & - & - & 19 & 5 & 7 & 35 \\
\hline & Hippodamia convergens (Guèrin-Menév.) & 2 & - & - & - & - & - & 3 & 5 \\
\hline & Coleomegilla maculata (DeGeer) & 2 & 1 & - & - & 1 & 2 & 5 & 11 \\
\hline & Olla v-nigrum (Mulsant) & - & - & 3 & - & - & - & - & 3 \\
\hline \multirow[t]{3}{*}{ Diptera } & Euxesta eluta (Loew) & - & - & - & - & - & - & 1 & 1 \\
\hline & Bemisa tabaci (Gennadius) & - & - & - & 1 & - & - & - & 1 \\
\hline & Ceresa brunnicornis (Linnaeus) & 16 & - & - & - & - & - & - & 16 \\
\hline \multirow{4}{*}{ Hemiptera } & Leptoglossus zonatus (Dallas) & 3 & - & 1 & - & - & - & 1 & 5 \\
\hline & Edessa meditabunda (Fabricius) & - & - & - & - & - & - & 1 & 1 \\
\hline & Uroleucon ambrosiae (Thomas) & 11 & - & - & - & 15 & 7 & 51 & 84 \\
\hline & Geocoris sp. (Fallen) & - & - & - & - & 2 & - & - & 2 \\
\hline \multirow{4}{*}{ Hymenoptera } & Acromyrmex coronatus (Fabricius) & 1 & 3 & 1 & 1 & - & - & - & 6 \\
\hline & Solenopis sp. (Westwood) & - & 1 & - & 1 & - & - & - & 2 \\
\hline & Apis mellifera (Linnaeus) & - & - & - & - & 2 & - & - & 2 \\
\hline & Trigona spinipes (Fabricius) & - & - & - & - & 1 & - & - & 1 \\
\hline Lepidoptera & Spodoptera frugiperda (J.E. Smith) & 1 & - & - & - & - & - & - & 1 \\
\hline Neuroptera & Chrysoperla sp. (Steinman) & - & 1 & - & - & - & - & 1 & 2 \\
\hline Total & & 45 & 12 & 17 & 8 & 57 & 21 & 72 & 232 \\
\hline
\end{tabular}


Tabela 2 - Análise faunística de insetos-pragas, coletados próximos à área cultivada com milho nas plantas hospedeiras, localizadas à distancia de: $\mathrm{T}_{1}$ Coniza sp. $(2 \mathrm{~m}) ; \mathrm{T}_{2}$ C. echinatus $(1 \mathrm{~m}) ; \mathrm{T}_{3}$ Amaranthus spp. $(2,8 \mathrm{~m}) ; \mathrm{T}_{4}$ E. heterophylla $(2,5 \mathrm{~m}) ; \mathrm{T}_{5} R$. raphanistrum $(2,3 \mathrm{~m}) ; \mathrm{T}_{6}$ B. pilosa $(2,6 \mathrm{~m})$ e $\mathrm{T}_{7}$ S. oleraceus $(1,6 \mathrm{~m})$ de área com cultivo. Bandeirantes, $\mathrm{PR}$.

\begin{tabular}{|c|c|c|c|c|c|c|c|}
\hline \multirow{2}{*}{ Índices } & \multicolumn{7}{|c|}{ Plantas hospedeiras } \\
\hline & $\mathrm{T}_{1}$ & $\mathrm{~T}_{2}$ & $\mathrm{~T}_{3}$ & $\mathrm{~T}_{4}$ & $\mathrm{~T}_{5}$ & $\mathrm{~T}_{6}$ & $\mathrm{~T}_{7}$ \\
\hline $\mathbf{S}$ & 7 & 4 & 3 & 4 & 4 & 2 & 5 \\
\hline$\alpha$ & 1,65 & 1,54 & 0,76 & 1,44 & 0,87 & 0,40 & 1,00 \\
\hline $\mathbf{H}$ & 1,50 & 1,28 & 0,51 & 1,07 & 0,95 & 0,68 & 0,40 \\
\hline $\mathbf{E}$ & 0,77 & 0,92 & 0,46 & 0,77 & 0,69 & 0,98 & 0,25 \\
\hline
\end{tabular}

$\mathrm{S}=$ Riqueza de espécies; $\alpha$ = Índice de Margalef; $\mathrm{H}$ = Índice de Shannon-Wiener; $\mathrm{E}=$ Equitabilidade.

Tabela 3 - Análise faunística de inimigos naturais, coletados próximos à área cultivada com milho nas plantas hospedeiras, localizadas à distancia de: $\mathrm{T}_{1}$ Coniza sp. $(2 \mathrm{~m}) ; \mathrm{T}_{2}$ C. echinatus $(1 \mathrm{~m}) ; \mathrm{T}_{3}$ Amaranthus spp. $(2,8 \mathrm{~m}) ; \mathrm{T}_{4}$ E. heterophylla $(2,5 \mathrm{~m}) ; \mathrm{T}_{5} R$. raphanistrum $(2,3 \mathrm{~m}) ; \mathrm{T}_{6}$ B. pilosa $(2,6 \mathrm{~m})$ e $\mathrm{T}_{7}$ S. oleraceus $(1,6 \mathrm{~m})$, de área com cultivo. Bandeirantes, $\mathrm{PR}$.

\begin{tabular}{|c|c|c|c|c|c|c|c|}
\hline \multirow{2}{*}{ Índices } & \multicolumn{7}{|c|}{ Plantas hospedeiras } \\
\hline & $\mathrm{T}_{1}$ & $\mathrm{~T}_{2}$ & $\mathrm{~T}_{3}$ & $\mathrm{~T}_{4}$ & $\mathrm{~T}_{5}$ & $\mathrm{~T}_{6}$ & $\mathrm{~T}_{7}$ \\
\hline $\mathbf{S}$ & 3 & 4 & 1 & 0 & 4 & 3 & 4 \\
\hline$\alpha$ & 1,03 & 1,86 & 0,00 & - & 0,96 & 0,91 & 1,08 \\
\hline $\mathbf{H}$ & 1,08 & 1,33 & 0,00 & - & 0,64 & 1,00 & 1,21 \\
\hline $\mathbf{E}$ & 0,98 & 0,96 & - & - & 0,46 & 0,90 & 0,87 \\
\hline
\end{tabular}

$\mathrm{S}=$ Riqueza de espécies; $\alpha$ = Índice de Margalef; $\mathrm{H}$ = Índice de Shannon-Wiener; $\mathrm{E}=$ Equitabilidade.

Tabela 4. Número total de espécies (t) de insetos, coletados próximo à área cultivada com algodão nas plantas hospedeiras, localizadas à distância de: T8 D. insularis $(5,5 \mathrm{~m})$; T9 P. maximum $(25 \mathrm{~m}) ;$ T10 Sida sp. $(5,8 \mathrm{~m}) ;$ T11 P. hysterophorus $(5,9 \mathrm{~m}) ; \mathrm{T} 12$ L. sibiricus $(9,5 \mathrm{~m})$ e T13 C. benghalensis $(10,5 \mathrm{~m})$ de área com cultivo. Bandeirantes, PR.

\begin{tabular}{|c|c|c|c|c|c|c|c|c|}
\hline \multirow{2}{*}{ Ordem } & \multirow{2}{*}{ Insetos } & \multicolumn{6}{|c|}{ Plantas hospedeiras } & \multirow[b]{2}{*}{$\mathrm{T}$} \\
\hline & & $\mathrm{T}_{8}$ & $\mathrm{~T}_{9}$ & $\mathrm{~T}_{10}$ & $\mathrm{~T}_{11}$ & $\mathrm{~T}_{12}$ & $\mathrm{~T}_{13}$ & \\
\hline \multirow[t]{9}{*}{ Coleoptera } & Astylus variegatus (Germar) & - & - & 1 & 12 & 1 & - & 14 \\
\hline & Diabrotica speciosa (Germar) & 13 & - & 6 & 11 & 14 & 3 & 47 \\
\hline & Epicauta atomaria (Gemar) & - & - & - & - & 1 & - & 1 \\
\hline & Lagria villosa (Fabricius) & - & 1 & - & - & - & 2 & 3 \\
\hline & Lebia concinna (Brullé) & - & - & - & - & - & 1 & 1 \\
\hline & Olla v-nigrum (Mulsant) & - & - & - & 2 & - & - & 2 \\
\hline & Cycloneda sanguinea (Linnaeus) & 1 & 1 & - & 1 & 1 & - & 4 \\
\hline & Eriopis connexa (Germar) & - & - & - & 2 & - & - & 2 \\
\hline & Hippodamia convergens (Guèrin-Mennévile) & 1 & - & 1 & 3 & - & - & 5 \\
\hline Diptera & Euxesta eluta (Loew) & - & 1 & 1 & - & - & - & 2 \\
\hline \multirow[t]{8}{*}{ Hemiptera } & Bemisa tabaci (Gennadius) & 1 & - & - & - & - & 2 & 3 \\
\hline & Dysdercus ruficollis (Linnaeus) & 15 & - & 29 & 4 & 2 & - & 50 \\
\hline & Thyanta perditor (Fabricius) & - & - & - & - & - & 3 & 3 \\
\hline & Piezodorus guildinii (Westwood) & - & - & - & - & - & 1 & 1 \\
\hline & Oebalus ypsilongriseus (De Geer) & 1 & - & 2 & 2 & - & - & 5 \\
\hline & Euschistus heros (Fabricius) & - & 8 & - & - & 2 & - & 10 \\
\hline & Leptoglossus zonatus (Dallas) & 2 & 1 & - & 2 & 2 & 5 & 12 \\
\hline & Empoasca sp. (Goethe) & 2 & - & - & - & - & 1 & 3 \\
\hline \multirow[t]{3}{*}{ Hymenoptera } & Acromyrmex coronatus (Fabricius) & - & 2 & - & 6 & - & 1 & 9 \\
\hline & Polybia sp.(Lepeletier) & - & 1 & - & - & - & - & 1 \\
\hline & Apis mellifera (Linnaeus) & - & - & - & 3 & 10 & - & 13 \\
\hline \multirow[t]{2}{*}{ Neuroptera } & Chrysoperla externa (Hagen) & - & 3 & - & - & - & - & 3 \\
\hline & Total & 36 & 18 & 40 & 48 & 33 & 19 & 194 \\
\hline
\end{tabular}

De modo geral, os índices $\alpha$ nos tratamentos foram baixos; de 0,40 a 1,65. Segundo Margalef (1972) esse índice normalmente varia entre 1,5 a 3,5 . A planta Coniza sp. destacou-se quanto à riqueza e diversidade de insetos-praga (Tabela 2), indicando predominância sobre as outras plantas hospedeiras. Quanto aos inimigos naturais observa-se que é nítida a superioridade de $C$. echinatus visto que abrigou a maior diversidade, particularmente representada pelas Coccinelidae (Tabela 3).

Próximo à área cultivada com algodão foram coletados 194 insetos, representados principalmente pela ordem Hemiptera e Coleoptera (Tabela 4). Destacaram-se como pragas o percevejo Pyrhocoridae Dysdercus ruficollis (L.) e o desfolhador D. speciosa (Tabela 4). 
Presente em quatro plantas espontâneas, D. ruficollis se destacou pelo seu elevado número na malvácea Sida sp. (Tabela 4). Este percevejo é considerado praga da cultura do algodão pois ao se alimentar provoca queda de botões florais, flores e maçãs novas e as maçãs mais desenvolvidas crescem defeituosas (GALLO et al, 2002; SANTOS, 2015). As espécies do gênero Dysdercus tem preferência alimentar por malváceas e são capazes de se reproduzir durante o ano todo em hospedeiros alternativos (FUJIHARA et al., 2011).

O conhecimento de hospedeiros alternativos é um dos aspectos importantes no controle de pragas, estes refúgios permitem a continuidade do ciclo da praga no campo durante várias gerações ao longo do ano (FORTI, 1990).

Onde há abundância de plantas hospedeiras o ambiente torna-se favorável para permanência de insetos herbívoros em maior diversidade; isto pode funcionar como fontes alternativa de alimento para os inimigos naturais generalistas permanecendo no campo nas épocas em que a população da praga principal está baixa (VANDERMEER, 1990; ANDOW, 1991).
A comunidade de insetos em $C$. bengalensis apresentou os maiores índices de riqueza, diversidade e equitabilidade de espécies os quais significam uma melhor distribuição do número de indivíduos das espécies de insetos coletados comparado às outras plantas (Tabela 5). No manejo de pragas do algodoeiro as plantas Sida santaremnensis, Sidastrum micranthum e Commelina bengalensis é bom que sejam eliminadas pois são hospedeiras de insetos vetores de doenças constituindo-se em potenciais reservatórios de vírus (MIRANDA et al., 2008).

A riqueza de espécies de inimigos naturais foi muito similar entre as plantas hospedeiras (Tabela 6), indicando que provavelmente a escolha do habitat pelos predadores alem de estar relacionada aos hábitos alimentares pode estar associada às características vegetais e preferência por certos estratos da planta.

A Coccinella septempunctata que escolhe ovipositar em plantas herbáceas infestadas por pulgões (IPERTI, 1999). Guedes (2013) relata a associação de joaninhas dos gêneros Adalia, Oenopia e Calvia com afídeos que ocorrem em arvores.

Tabela 5. Análise faunística de insetos pragas, coletados próximos à área cultivada com algodão nas plantas hospedeiras, localizadas à distância de: $\mathrm{T}_{8} D$. insularis $(5,5 \mathrm{~m}) ; \mathrm{T}_{9} P$. maximum $(25 \mathrm{~m}) ; \mathrm{T}_{10}$ Sida $\mathrm{sp} .(5,8 \mathrm{~m}) ; \mathrm{T}_{11}$ P. hysterophorus $(5,9 \mathrm{~m}) ; \mathrm{T}_{12} L$. sibiricus $(9,5 \mathrm{~m})$ e $\mathrm{T}_{13}$ C. benghalensis $(10,5 \mathrm{~m})$ de área com cultivo. Bandeirantes, $\mathrm{PR}$.

\begin{tabular}{|c|c|c|c|c|c|c|}
\hline \multirow{2}{*}{ Índices } & \multicolumn{6}{|c|}{ Plantas hospedeiras } \\
\hline & $\mathrm{T}_{8}$ & $\mathrm{~T}_{9}$ & $\mathrm{~T}_{10}$ & $\mathrm{~T}_{11}$ & $\mathrm{~T}_{12}$ & $\mathrm{~T}_{13}$ \\
\hline$S$ & 6 & 5 & 5 & 6 & 6 & 8 \\
\hline$\alpha$ & 1,42 & 1,56 & 1,09 & 1,38 & 1,62 & 2,42 \\
\hline $\mathrm{H}$ & 1,27 & 1,18 & 0,84 & 1,58 & 1,22 & 1,92 \\
\hline E & 0,71 & 0,73 & 0,52 & 0,88 & 0,68 & 0,92 \\
\hline
\end{tabular}

$\mathrm{S}=$ Riqueza de espécies; $\alpha=$ Índice de Margalef; $\mathrm{H}=$ Índice de Shannon-Wiener; E = Equitabilidade.

Tabela 6. Análise faunística de inimigos naturais, coletados próximos à área cultivada com algodão nas plantas hospedeiras, localizadas à distancia de: $\mathrm{T}_{8}$ D. insularis $(5,5 \mathrm{~m}) ; \mathrm{T}_{9}$ P. maximum $(25 \mathrm{~m}) ; \mathrm{T}_{10}$ Sida sp. $(5,8 \mathrm{~m}) ; \mathrm{T}_{11}$ P. hysterophorus $(5,9 \mathrm{~m}) ; \mathrm{T}_{12} L$. sibiricus $(9,5 \mathrm{~m})$ e $\mathrm{T}_{13}$ C. benghalensis $(10,5 \mathrm{~m})$ de área com cultivo. Bandeirantes, $\mathrm{PR}$.

\begin{tabular}{|c|c|c|c|c|c|c|}
\hline \multirow{2}{*}{ Índices } & \multicolumn{6}{|c|}{ Plantas hospedeiras } \\
\hline & $\mathrm{T}_{8}$ & $\mathrm{~T}_{9}$ & $\mathrm{~T}_{10}$ & $\mathrm{~T}_{11}$ & $\mathrm{~T}_{12}$ & $\mathrm{~T}_{13}$ \\
\hline $\mathrm{S}$ & 2 & 3 & 1 & 4 & 1 & 1 \\
\hline$\alpha$ & 1,44 & 1,24 & - & 1,37 & - & - \\
\hline $\mathrm{H}$ & 0,69 & 0,95 & 0,00 & 1,28 & 0,00 & 0,00 \\
\hline $\mathrm{E}$ & 1,00 & 0,86 & - & 0,92 & - & - \\
\hline
\end{tabular}

$\mathrm{S}=$ Riqueza de espécies; $\alpha$ = Índice de Margalef; $\mathrm{H}=$ Índice de Shannon-Wiener; $\mathrm{E}=$ Equitabilidade.

\section{Conclusões}

Os insetos presentes em maior número nas plantas daninhas próximas à cultura do milho foram Uroleucon ambrosiae, Diabrotica speciosa, e Eriopis connexa. Nas plantas localizadas nas proximidades da área de cultivo de algodão os insetos em maior número foram Dysdercus ruficollis e Diabrotica speciosa.

A planta Coniza sp. abrigou a maior riqueza e diversidade de insetos-pragas; $C$. echinatus abrigou a maior diversidade de inimigos naturais, entre as hospedeiras localizadas próximo área de cultivo de milho. Entre as plantas próximas a área de cultivo de algodão, C. bengalensis apresentou os maiores índices faunísticos para insetos-pragas; $P$. hysterophorus foi a mais rica em espécies de inimigos naturais.

\section{Referências Bibliográficas}

ALTIERI, M. A.; SILVA E. N.; NICHOLLS, C. I. O papel da biodiversidade no maneio de pragas. Ribeirão Preto-SP: Holos, 2003. 226 p.

ANDOW, D. A. Vegetational diversity and arthropod population response. Annual Review of Entomology, Palo Alto, v. 36, n. 1, p. 561-586, 1991. 
AUAD, A. M.; FREITAS, S.; BARBOSA, L. R. Potencial de alimentação de Chrysoperla externa (Hagen, 1861) (Neuroptera, Chrysopidae) em diferentes densidades de Uroleucon ambrosiae (Thomas, 1878) (Hemiptera, Aphididae). Revista Brasileira de Entomologia, São PauloSP, v. 47, n. 1, p.15-18, mar. 2003.

CARRANO-MOREIRA, A. F. Manejo Integrado de pragas florestais: fundamentos ecológicos, conceitos e táticas de controle. Rio de Janeiro-RJ: Technical Books. 2014, p. 218 222.

CORRÊA-FERREIRA, B. S. Amostragem de pragas da soja. In: HOFFMANN-CAMPO, C. B.; CORREAA-FERREIRA, B. S.; MOSCARDI, F. Soja: manejo integrado de insetos e outros artrópodes-praga. Brasília-DF: Embrapa Soja, 2012, v. 1, p. 631-672.

FAVERO, C.; JUCKSCH, I.; ALVARENGA, R. C.; COSTA, L. M. Modificação na população de plantas espontâneas na presença de adubos verdes. Pesquisa Agropecuária Brasileira, Brasília-DF, v. 36, n. 11, p. 1355-1362,2001.

FORTI, L. C. Ecologia no manejo integrado de pragas. In CROCOMO, W. B. (ed.). Manejo integrado de pragas. Botucatu-SP: CETESB, 1990. 350 p.

FUERST, E. P.; PUTNAN, A. R. Separating the competitive and allelopathic components of interference: theoretical principles. Journal of Chemical Ecology, New York, v. 9, n. 8, p. 937-944, 1983.

FUJIHARA, R. T.; FORTI, L. C.; ALMEIDA, M. C.; BALDIN, E. L. L (Eds.). Insetos de importância econômica: guia ilustrado para identificação de famílias. Botucatu-SP: FEPAF, 2011, 391 p.

GALLO, D.; NAKANO,O.; SILVEIRA NETO, S. S.; CARVALHO, R. P. L.; BATISTA ,G. C.; BERTIFILHO, E.; PARRA, J. R. P.; ZUCCHI, R. A.; ALVES, S. B.; VENDRAMIM, J. D.; MARCHINI, L. C.; LOPES, J. R. S.; OMOTO, C. Entomologia Agrícola. Piracicaba-SP: FEALQ, 2002. 920 p.

GUEDES, C. F. C. Preferência alimentar e estratégias de alimentação em Coccinellidae (Coleoptera). Oecologia Australis, Rio de Janeiro-RJ, v. 17, n. 2, p. 249-270, 2013.

GODOY, K. B.; ÁVILA, C. J.; DUARTE, M. M.; ARCE, C. C. M. Parasitismo e sítios de diapausa de adultos do percevejo marrom, Euschistus heros na região da Grande Dourados, MS. Ciência Rural, Santa Maria-RS, v. 40, n. 5, p. 11191202, 2010 .

HOFFMANN-CAMPO, C. B.; OLIVEIRA, L. J.; MOSCARDI, F.; CORREIA-FERREIRA, B. S.; CORSO, I. C. Pragas que atacam plântulas, hastes e pecíolos da soja. In: HOFFMANN-CAMPO C. B., CORRÊA-FERREIRA B. S., MOSCARDI F. Soja: manejo integrado de insetos e outros artrópodes-praga. Brasília-DF: Embrapa Soja, 2012. p. 145212.

IPERTI, G. Biodiversity of predaceous Coccinellidae in relation to bioindication and economic importance. Agriculture, Ecosystems and Environment, Amsterdam, v. 74, p. 323-342, 1999.

MACEDO, J. F.; MARTINS, R. P. Potencial da erva daninha Waltheria americana (Sterculiaceae) no Manejo Integrado de Pragas e Polinizadores: Visitas de Abelhas e Vespas. Anais da
Sociedade Entomológica do Brasil, Londrina-PR, v. 28, n. 1, p.29-40, 1998.

MARGALEF, R. Homage to Evelyn Hutchinson, or why is there an upper limit to diversity. In: DEEVEY, E. S. Growth by Intussusception: Ecological Essays in Honor of $G$. Evelyn. 44. ed. Hamden: Connecticut Academy of Arts and Sciences, 1972. p. 211-235.

MIRANDA, J. E.; SUASSUNA, N. D.; MORELLO, C. L.; SILVA, M. V. F.; FREIRE, E. C. Doença azul do algodoeiro: Novos aspectos a serem considerados no manejo. Campina Grande-PB: Embrapa Algodão, 2008. 12 p. (Circular Técnica, 121).

NDAKIDEMI, B.; MTEI, K.; NDAKIDEMI, P. A. The potential of common beneficial insects and strategies of maintaining them in bean fields of Sub Saharan Africa. American Journal of Plant Sciences, v. 7, p. 425-436, 2016.

PANIZZI, A. R.; BUENO, A. F.; SILVA, F. A. C. Insetos que atacam vagens e grãos. In: HOFFMANN-CAMPO C. B., CORRÊA-FERREIRA B. S., MOSCARDI F.. Soja: manejo integrado de insetos e outros artrópodes-praga. Brasília-DF: Embrapa Soja, 2012, p. 336- 420.

PINTO-COELHO, R. M. Fundamentos em ecologia. Porto Alegre-RS: Artmed, 2000. 225 p.

RICE, E. L. Allelopathy. New York: Academic Press, 1974. $333 \mathrm{p}$.

RICKLEFS, R. E. A. Economia da Natureza. 3. ed. Rio de Janeiro-RJ: Guanabara Koogan, 1996. 470 p.

SALVADORI, J. R.; ÁVILA, C. J.; SILVA, M. T. B.. Pragas de solo no Brasil. Passo Fundo-RS: Embrapa Trigo, 2004.541 p.

SANTOS, W. J. Manejo das pragas do algodoeiro, com destaque para o cerrado brasileiro. In: FREIRE, E. C. Algodão: no cerrado do Brasil. 3. ed. Brasília-DF: ABRAP, 2015, p. 267-364.

SILVA, R. B.; CRUZ, I.; ZANUNCIO, J. C.; FIGUEIREDO, M. L. C.; CANEVARI, G. C.; PEREIRA, A. G.; SERRÃO, J. E. Biological aspects of Eriopis connexa (Germar) (Coleoptera: Coccinellidae) fed on different insect pests of maize (Zea mays L.) and sorghum [Sorghum bicolor L. (Moench.)] Brazilian Journal of Biology, São Carlos-SP, v. 73, n. 2, p. 419-424, 2013.

SILVEIRA NETO, S.; NAKANO, O.; BARBIN, D.; VILLA NOVA, N. A. Manual de Ecologia dos Insetos. São PauloSP: Agronômica Ceres, 1976. 419p.

SOUTHWOOD, T. R. E. Ecological methods: with particular reference to the study of insect populations. 2. ed. London: Chapman \& Hall, 1995. 524 p.

THEBAUD, C.; ABBOTT, R. J. Characterization of invasive Conyza species (Asteraceae) in Europe: quantitative trait and isozyme analysis. American Journal of Botany, Columbus, v. 82 , n. 3, p. $360-368,1995$.

VANDERMEER, J. H. Intercropping. In: CARROL, C. R.; VANDERMEER, J. H.; ROSSET, P. M. (Ed.). Agroecology. New York: McGraw-Hill, 1990. p. 481-516. 